\title{
Caracterização Sociodemográfica e Avaliação da Qualidade de Vida em Mulheres com Câncer de Mama em Tratamento Quimioterápico Adjuvante
} Sociodemographic Characterization and Evaluation of Quality of Life in Women with Breast Cancer Undergoing Adjuvant Chemotherapy Caracterización Sociodemográfica y Evaluación de la Calidad de Vida en Mujeres con Cáncer de Mama Sometidas a Quimioterapia Adyuvante

\author{
Audir Giordano C. Guimarães'; Anna Cláudia Y. dos Anjos
}

\section{Resumo}

Introdução: A avaliação da qualidade de vida $(\mathrm{QV})$ é importante auxiliar da prática clínica. Objetivo: Avaliar a QV em mulheres com câncer de mama e caracterizá-las quanto aos aspectos sociodemográficos durante o tratamento quimioterápico adjuvante. Método: Estudo descritivo-exploratório, com abordagem quantitativa. Foram utilizados dois instrumentos: um para a avaliação sociodemográfica e outro para avaliação da QV (EORTC QLQ-C30). A amostra constou de 14 pacientes atendidas em ambulatório de oncologia de um hospital universitário de Minas Gerais, de abril a outubro de 2011. Resultados: A maioria da população estudada possui cor da pele branca (57,1\%), é casada (64,3\%), havendo predomínio de mulheres com nível fundamental incompleto $(35,7 \%)$ e médio completo $(35,7 \%)$. A renda familiar mensal variou de dois a seis salários mínimos (78,6\%). Quanto à procedência, 50\% são de Uberlândia e $50 \%$ de fora dessa cidade. Quanto ao esquema terapêutico, foi predominante a utilização do FAC (50\%), seguido do esquema CMF (28,6\%). Ao avaliar isoladamente os domínios função física, emocional e cognitiva, verificou-se piora na QV do início para o final do tratamento. Em relação aos aspectos físicos, todas as mulheres apresentaram piora da QV. Na avaliação qualidade de vida/medida geral de saúde (QV/MGS), foi verificada discreta redução, mesmo com avaliação positiva das pacientes durante todo estudo. Conclusáo: $\mathrm{O}$ conjunto de resultados mostrou características da amostra semelhantes à de outros estudos, além de evidenciar que, a despeito de todos os benefícios cientificamente comprovados do tratamento quimioterápico adjuvante, observa-se seu impacto negativo sobre a QV, durante todo o período desse tratamento.

Palavras-chave: Qualidade de Vida; Neoplasias da Mama; Mulheres; Quimioterapia Adjuvante; Fatores Socioeconômicos; Inquéritos Demográficos

\footnotetext{
Trabalho de Pesquisa apresentado ao Programa de Residência Multiprofissional de Uberlândia, Hospital de Clínicas da Universidade Federal de Uberlândia (UFU). Uberlândia (MG), Brasil, 2011.

${ }^{1}$ Enfermeiro Graduado pela UFU e Residente em Oncologia do Programa de Residência Multiprofissional HC/UFU. E-mail: guimaraes555@yahoo.com.br. Uberlândia (MG), Brasil. Pesquisador.

${ }^{2}$ Enfermeira. Doutora em Enfermagem. Professora Adjunta do Curso de Graduação em Enfermagem e Coordenadora do Programa de Residência Multiprofissional Atenção em Oncologia da UFU. Orientadora da Pesquisa.

Endereço para correspondência: Anna Cláudia Y. dos Anjos. E-mail: annaclaudia1971@gmail.com.
} 


\section{INTRODUÇÃO}

O Instituto Nacional de Câncer José Alencar Gomes da Silva (INCA) define câncer como um crescimento desordenado das células que podem invadir tecidos e órgãos e que ainda pode se espalhar para outras regiốes do corpo (metástases) ${ }^{1}$.

Atualmente, o câncer de mama é o que mais acomete as mulheres brasileiras e, além disso, é o mais temido no grupo feminino, visto que provoca forte impacto físico e psicológico, envolvendo negativamente a percepção da sexualidade e a autoimagem, mais do que se observa em qualquer outro tipo de câncer ${ }^{2}$.

Em um primeiro momento, um diagnóstico como o do câncer de mama certamente causa um efeito devastador na vida de uma mulher. Aos poucos e, sobretudo, após a fase aguda do tratamento, há uma tendência a se retornar à qualidade de vida $(\mathrm{QV})$ de antes do diagnóstico e tratamento, ou mesmo ocorrem mudanças positivas na QV, com oportunidade de crescimento pessoal. O enfrentamento da doença leva, em alguns casos, a um maior e melhor sentido para a vida e à reestruturaçáo de prioridades ${ }^{3}$.

O câncer, em sua fase inicial, pode ser controlado e curado cirurgicamente, associado ou nấo ao uso de quimioterápicos e/ou radioterapia. $\mathrm{O}$ advento da quimioterapia e novas técnicas de radioterapia vieram contribuir para o tratamento microscópico, o que permitiu, nos últimos anos, uma nova abordagem na extensão da cirurgia para o câncer ${ }^{1}$.

O tratamento do câncer com a utilização de quimioterápicos pode ter objetivos curativos ou paliativos. A quimioterapia representa um avanço na cura e controle do câncer, aumentando a expectativa e QV do paciente. É essencial, no entanto, que os profissionais de saúde que atuam nessa área tornem efetiva a orientaçáo quanto aos objetivos e efeitos colaterais do tratamento quimioterápico, além, sobretudo, de oferecer apoio emocional ${ }^{4}$.

A maioria dos quimioterápicos antineoplásicos atua de forma não específica, lesando tanto células malignas quanto benignas. Como as diferenças entre as duas populaçôes celulares são mais quantitativas do que qualitativas, uma linha muito tênue separa o sucesso terapêutico de uma toxicidade inaceitável ${ }^{5}$.

Como modalidade de tratamento do câncer de mama, a quimioterapia tem a sua finalidade estabelecida, seja em caráter neoadjuvante, adjuvante ou paliativo. A quimioterapia adjuvante se caracteriza por ser administrada após a cirurgia curativa, período em que o paciente está aparentemente sem doença, mas quando ainda existe risco de recidiva. A quimioterapia adjuvante retarda o surgimento de metástases e melhora os índices de cura, influenciando por sua vez a $\mathrm{QV}^{6}$.
A detecção precoce do câncer, o avanço no tratamento, o número crescente de sobreviventes e a maior importância atribuída aos fatores psicossociais têm levado a maior ênfase para a QV das pessoas com câncer ${ }^{7}$.

QV é um termo de difícil definição, pois inclui uma variedade potencial de condiçóes que afetam a percepção do indivíduo, seus sentimentos e comportamentos relacionados ao seu funcionamento diário, incluindo a sua condição de saúde e intervenções médicas. A oncologia é uma das áreas que mais tem avaliado a $\mathrm{QV}$, pois os tratamentos, por vezes agressivos, apesar de acrescentarem "anos à vida", em geral não acrescentam "vida aos anos".

A Organização Mundial da Saúde (OMS) define QV como:

a percepção do indivíduo de sua posiçáo na vida, no contexto da cultura, sistema de valores nos quais ele vive em relação aos seus objetivos, expectativas, padróes e preocupaçóes.

Qualidade de vida relacionada à saúde (QVRS) e estado subjetivo de saúde são conceitos afins, centrados na avaliação subjetiva do paciente, mais necessariamente ligados ao impacto do estado de saúde sobre a capacidade do indivíduo viver plenamente' ${ }^{9}$. Os resultados da avaliação da QV são importantes auxiliares da prática clínica, além de ajudar o paciente a identificar as necessidades para adaptaçóes $^{10}$.

O objetivo deste estudo foi avaliar a QV em mulheres com câncer de mama e caracterizá-las quanto aos aspectos sociodemográficos, durante o tratamento quimioterápico adjuvante.

\section{MÉTODO}

A avaliação da QV de pacientes com diagnósticos de câncer de mama em tratamento quimioterápico foi realizada por meio de um estudo descritivo-exploratório, com abordagem quantitativa em que foram utilizados dois instrumentos, sendo um para a avaliação sociodemográfica e outro (EORTC QLQ-C30) para avaliação da QV.

A populaçáo estudada foi composta por 14 mulheres com idade superior a 18 anos, que participavam de um projeto de extensão, diagnosticadas com câncer de mama com indicaçáo de tratamento quimioterápico adjuvante e atendidas em ambulatório de oncologia de um Hospital Universitário do interior de Minas Gerais, durante o período de abril a outubro de 2011.

O projeto foi aprovado pelo Comitê de Ética em Pesquisa (CEP) da Universidade Federal de Uberlândia (UFU) sob o número 016/11. As mulheres foram convidadas a participar da pesquisa, no dia em que foi estabelecido o tratamento, assinando, previamente à coleta 
de dados, o Termo de Consentimento Livre e Esclarecido (TCLE). Não foram incluídas no estudo mulheres com indicação de tratamentos neoadjuvante e neoadjuvante combinados ou mulheres em cuidados paliativos.

As pacientes foram inicialmente avaliadas de acordo com as características sociodemográficas, por meio da aplicação do questionário A (Anexo 1). Posteriormente, para a avaliação da $\mathrm{QV}$, utilizou-se o instrumento, já validado no Brasil, questionário B (Anexo 2) European Organization for Research and Treatment of Cancer Breast Cancer-Specific of Life Questionnaire (EORTC QLQ-C30), sendo aplicado às pacientes, em forma de entrevista, no primeiro, terceiro e sexto ciclos de tratamento. Os domínios contemplados pelo instrumento EORTC QLQ-C30 são: função fisicica, função emocional, função cognitiva, função social, escala de sintomas e QV/medida geral de saúde (QV/MGS). Nesse instrumento, quanto maior for o valor do escore médio apresentado nos domínios função física, emocional, cognitiva e social, melhor é a QV. Já na escala de sintomas, quanto maior for o valor do escore médio apresentado, mais intenso é o sintoma; porém, quanto menor o valor do escore médio, menor é a intensidade do sintoma apresentado pela paciente, indicando, desde então, melhor QV.

A análise estatística foi realizada por meio do programa SPSS 11.5. As variáveis contínuas que apresentaram distribuição normal foram expressas em média, desviopadrão e valores mínimos e máximos das pontuações de cada pergunta do EORTC QLQ-C30. A análise descritiva foi apresentada em tabelas de distribuiçáo de frequência e medidas descritivas, como média e desvio-padrão, para variáveis quantitativas.

\section{RESULTADOS E DISCUSSÃO}

As variáveis sociodemográficas (faixa etária, cor da pele, escolaridade, estado civil e procedência) e clínicas abordadas neste estudo estáo apresentadas na Tabela 1. Em relação à variável econômica, foi utilizada a renda familiar e, quanto às variáveis clinicas, foi abordado o esquema quimioterápico.

A faixa etária das participantes apresentou predominância acima de 46 anos (57,2\%). Esses dados eram esperados, uma vez que estudos confirmam não ser frequente a ocorrência de câncer de mama em mulheres com menos de 40 anos $^{11,12,13}$.

Apesar de ser uma condição incomum em mulheres com menos de 40 anos idade, o câncer de mama tem sido observado em um número cada vez maior de mulheres jovens, principalmente naquelas com história familiar positiva de carcinoma mamário pré-menopausa e fatores genéticos de risco ${ }^{11}$. Avaliando os aspectos epidemiológicos da doença em mulheres jovens, pesquisadores observaram que $(5,5 \%)$ das pacientes apresentaram câncer de mama antes dos 35 anos de idade ${ }^{12}$. Considerando essa mesma faixa etária, em outro estudo ${ }^{13}$, constatou-se que $(12,5 \%)$ das pacientes apresentaram o tumor antes dos 40 anos de idade e $(31,8 \%)$ antes dos 45 anos.

Em relação à cor da pele, $57,1 \%$ das participantes classificaram-se como brancas e $41,9 \%$ como negras (Tabela 1). A prevalência e suscetibilidade de surgimento de neoplasias de mama são maiores nas mulheres de pele branca; porém o diagnóstico tardio é realizado mais frequentemente na população feminina afrodescendente. Neste sentido, faz-se necessário contemplar o atendimento a todas mulheres, independentemente de raça ou cor. No campo da saúde, há evidências de vulnerabilidades que fragilizam mulheres negras em termos de diferenças de morbidade e acesso à saúde, principalmente na qualidade dos serviços ginecológicos e obstétricos ${ }^{14}$.

Nessas pacientes, os níveis de escolaridade predominantes são fundamental incompleto $(35,7 \%)$ e nível médio completo $(35,7 \%)$, evidenciando ainda que $21,4 \%$ das mulheres com nível fundamental incompleto possuem mais de 60 anos e 14,3\% das mulheres com nível médio completo possuem de 36 a 45 anos. Tais resultados permitiram inferir que, quanto maior a idade menor o nível de escolaridade e quanto mais jovem a paciente, maior o nível de escolaridade. Percebe-se, ainda, uma percentagem baixa $(14,1 \%)$ das pacientes com nível superior, mostrando que as pacientes atendidas nessa Instituição Pública de Saúde possuem menor nível educacional (85,7\%). Devese considerar que a escolaridade representa um fator importante no planejamento e implementação de atividades educativas realizadas pelo enfermeiro, uma vez que essas devem-se adequar à linguagem de fácil compreensão, facilitando a comunicação e visando ao entendimento e à participação das mulheres com câncer de mama nas orientações propostas ${ }^{15}$.

Estudando a relação entre o tratamento quimioterápico e dados sociodemográficos e econômicos em pacientes com diagnóstico de câncer de mama, utilizando instrumentos que avaliam a QV, o EORT QLQ-C30 e o questionário específico para câncer de mama, o QLQ BR-23, pesquisadores ${ }^{16}$ verificaram que os escores de QV em pacientes submetidas à quimioterapia estiveram baixos nas quatros dimensôes que os instrumentos se propóem a avaliar, com uma desproporcionalidade de grande impacto naquelas com baixo nível de educação. Tais pesquisadores concluíram ainda que se deve reconhecer o impacto do tratamento na QV e que este pode variar de acordo com a idade e o nível educacional das mulheres. Considerando portanto a referência anteriormente feita ${ }^{9}$, em que a $\mathrm{QV}$ deve ser avaliada sob a perspectiva do paciente, salienta-se aqui o quanto o impacto dessa modalidade de tratamento pode de fato ser significativa, requerendo pronta e eficaz intervenção da equipe de saúde. 
Tabela 1. Características sociodemográficas e clínicas em relação à faixa etária de 14 pacientes com diagnóstico de câncer de mama e submetidos à quimioterapia adjuvante, no Hospital de Clínicas da Universidade Federal de Uberlândia. Setor de Oncologia, abril a outubro de 2011

\begin{tabular}{|c|c|c|c|c|c|c|c|c|c|c|}
\hline \multirow{3}{*}{$\begin{array}{l}\text { Variáveis } \\
\text { demográficas }\end{array}$} & \multicolumn{8}{|c|}{ Faixa etária } & \multirow{2}{*}{\multicolumn{2}{|c|}{ Total }} \\
\hline & \multicolumn{2}{|c|}{15 a 35 anos } & \multicolumn{2}{|c|}{36 a 45anos } & \multicolumn{2}{|c|}{46 a 59 anos } & \multicolumn{2}{|c|}{$\begin{array}{c}\text { Acima de } 60 \\
\text { anos }\end{array}$} & & \\
\hline & $\mathbf{F}$ & $\%$ & $\mathbf{F}$ & $\%$ & $\mathbf{F}$ & $\%$ & $\mathbf{F}$ & $\%$ & $\mathbf{F}$ & $\%$ \\
\hline \multicolumn{11}{|l|}{ Cor } \\
\hline Branca & 1 & 7,1 & 2 & 14,3 & 2 & 14,3 & 3 & 21,4 & 8 & 57,1 \\
\hline Negra & 2 & 14,3 & 1 & 7,1 & 2 & 14,3 & 1 & 7,1 & 6 & 42,9 \\
\hline \multicolumn{11}{|l|}{ Escolaridade } \\
\hline Fundamental & 0 & 0 & 1 & 7,1 & 1 & 7,1 & 3 & 21,4 & 5 & 35,7 \\
\hline incompleto & 2 & 14,3 & 0 & 0 & 1 & 7,1 & 0 & 0 & 2 & 14,3 \\
\hline Médio incompleto & 1 & 7,1 & 2 & 14,3 & 1 & 7,1 & 1 & 7,1 & 5 & 35,7 \\
\hline Médio completo & 1 & 7,1 & 1 & 7,1 & 0 & 0 & 0 & 0 & 1 & 7,1 \\
\hline Superior completo & 0 & 0 & 0 & 0 & 1 & 7,1 & 0 & 0 & 1 & 7,1 \\
\hline Superior incompleto & 0 & 0 & 0 & 0 & 0 & 0 & 0 & 0 & 0 & 0 \\
\hline \multicolumn{11}{|l|}{ Estado civil } \\
\hline Casada & 2 & 14,3 & 1 & 7,1 & 3 & 21,4 & 3 & 21,4 & 9 & 64,3 \\
\hline Solteira & 1 & 7,1 & 0 & 0 & 1 & 7,1 & 1 & 7,1 & 3 & 21,4 \\
\hline Divorciada/separada & 0 & 0 & 1 & 7,1 & 0 & 0 & 0 & 0 & 1 & 7,1 \\
\hline Viúva & 0 & 0 & 1 & 7,1 & 0 & 0 & 0 & 0 & 1 & 7,1 \\
\hline \multicolumn{11}{|l|}{ Renda familiar } \\
\hline Até 1 salário mínimo & 0 & 0 & 1 & 7,1 & 0 & 0 & 1 & 7,1 & 2 & 14,3 \\
\hline $\begin{array}{l}\text { De } 2 \text { a } 6 \text { salários } \\
\text { mínimos }\end{array}$ & 2 & 14,3 & 2 & 14,3 & 4 & 28,6 & 3 & 21,4 & 11 & 78,6 \\
\hline $\begin{array}{l}\text { De } 6 \text { a } 10 \text { salários } \\
\text { mínimos }\end{array}$ & 1 & 7,1 & 0 & 0 & 0 & 0 & 0 & 0 & 1 & 7,1 \\
\hline \multicolumn{11}{|l|}{ Procedência } \\
\hline Uberlândia & 2 & 14,3 & 1 & 7,1 & 3 & 24,4 & 1 & 7,1 & 7 & 50 \\
\hline Outras cidades & 1 & 7,1 & 2 & 14,3 & 1 & 7,1 & 3 & 21,4 & 7 & 50 \\
\hline \multicolumn{11}{|l|}{ Esquema terapêutico } \\
\hline FAC + TXT & 0 & 0 & 0 & 0 & 1 & 7,1 & 0 & 0 & 1 & 7,1 \\
\hline CMF & 1 & 7,1 & 1 & 7,1 & 1 & 7,1 & 1 & 7,1 & 4 & 28,6 \\
\hline FAC & 2 & 14,3 & 2 & 14,3 & 0 & 0 & 3 & 21,4 & 7 & 50,0 \\
\hline OUTROS & 0 & 0 & 0 & 0 & 2 & 14,3 & 0 & 0 & 2 & 14,3 \\
\hline
\end{tabular}

Quanto à situação conjugal das pacientes entrevistadas, houve predominância de casadas (9) (64,3\%) em todas as faixas etárias, com maior concentração acima de 45 anos. Os resultados também mostram que $21,4 \%$ são solteiras, as demais são divorciadas $(7,1 \%)$ e viúvas $(7,1 \%)$.

A Tabela 1 mostra também que 11 pacientes $(78,6 \%)$ ganham entre dois a seis salários mínimos e apenas 14,3\% ganham menos de um salário mínimo. Esses dados não são compatíveis com a situaçáo econômica da maioria da população brasileira, uma vez que dados do $\mathrm{IBGE}^{17}$ revelam a distribuição percentual de rendimento mensal familiar das grandes regióes, unidades da federação e regióes metropolitanas, onde $51,3 \%$ ganham menos de um salário mínimo, e 23,2\% ganham de um a dois salários mínimos.
Verificou-se, neste estudo, que a procedência das participantes foi de $50 \%$ de Uberlândia e $50 \%$ de outras cidades, por serem desprovidas de centros de atendimento de alta complexidade em suas cidades de origem e por Uberlândia ser caracterizada como cidade referência de saúde para alta complexidade de cidades vizinhas. Alguns estudos mostram que o fato de passarem maior tempo em rodovias, estressadas com congestionamentos e riscos de acidentes, faz com que essas mulheres que recebem tratamento fora de suas cidades sofram maior impacto em sua $\mathrm{QV}$ do que aquelas mulheres que residem no próprio município de tratamento ${ }^{18}$. A precariedade das estradas também é um fator importante que deve ser considerado, pois resulta em tempo prolongado de viagem, o que afetará diretamente a $\mathrm{QV}$ do viajante, devido ao cansaço provocado por longas horas em rodovias ${ }^{18}$. 
Quanto aos esquemas terapêuticos utilizados como tratamento adjuvante para o câncer de mama, nas 14 mulheres diagnosticadas, verificou-se que $50 \%$ delas foram tratadas com Fluorouracil + Adriblastina + Ciclofosfamida (FAC); $28,6 \%$ tiveram como terapia adjuvante o Ciclofosfamida + Metrotexato + Fluorouracil (CMF); 14,3\% apenas Docetaxel. Esses dados corroboram os achados na literatura que revelam, em consensos internacionais e na realidade nacional e latino-americana, recomendaçóes para utilização de quimioterapia adjuvante em pacientes pós-cirurgia com axila comprometida, dos esquemas FAC e FEC (5-Fluorouracil + Epirrubicina + Ciclofosfamida). É sabido que esses esquemas têm uma maior complexidade, toxicidade e alto custo, assim, por sua vez, geram maior ocorrência de efeitos indesejados, reaçóes adversas e consequentemente diminuição da $\mathrm{QV}^{19}$. A Tabela 2 mostra as médias e seus respectivos desvios-padrão de cada domínio do instrumento de QV EORTC QLQ-30, do total de pacientes participantes da pesquisa no início do primeiro, terceiro e último ciclos do tratamento quimioterápico adjuvante, representados pelas numeraçóes 1,2 e 3, respectivamente, em cada uma das dimensôes analisadas. Em relação às dimensôes do EORTC QLQ-C30, os valores médios dos escores encontrados variaram de 0 a 89,3.

Ao analisar essa tabela, verifica-se que o domínio função física apresentou redução dos valores médios dos escores no decorrer dos ciclos de tratamento, indicando piora na QV nesse domínio.

Quanto ao domínio função emocional, não há grandes variações entre os ciclos analisados: 66,9\%-65,0\%, mostrando uma pequena diminuição do escore de QV, ao final do tratamento. A função cognitiva apresentou queda nos valores médios dos escores durante os ciclos de quimioterapia analisados, traduzindo-se em piora da QV, uma vez que os escores apresentados foram respectivamente de $79,6 \%, 69,2 \%$ e $58,4 \%$.

Sobre o domínio função social, observa-se um aumento no valor médio do escore apresentado no segundo momento de análise (100\%), indicando melhora desse aspecto nessa fase do tratamento; porém com redução do valor médio do escore no último ciclo $(86,9 \%)$, o que significa oscilação da QV e forte influência do tratamento quimioterápico no domínio função social, entre as mulheres estudadas. Em relação à escala de sintomas, quando analisada a fadiga, foram percebidos menores valores médios dos escores no início do tratamento $(27,4 \%)$ havendo crescimento no terceiro ciclo $(40,5 \%)$ e no último ciclo $(41,7 \%)$; observa-se ainda que a variação apresentada nos escores médios da primeira para a segunda e terceira análise foi de quase 50\% de aumento, traduzindo portanto uma intensificação desse sintoma durante todo o tratamento.

Percebe-se, ainda, que o sintoma de fadiga já estava presente no início do tratamento em $27,4 \%$, havendo intensificação nos ciclos subsequentes. A ocorrência da fadiga é esperada com o tratamento quimioterápico, pois estudos mostram que a sensação de fadiga é experimentada por $72 \%$ a $95 \%$ de todos os pacientes durante e após o tratamento ${ }^{20}$. Pesquisadores apontaram que a fadiga pode ser a principal causa de impacto na QV desses pacientes ${ }^{21}$. Especificamente, a fadiga é um dos sintomas mais comuns em pacientes com câncer; outro estudo mostrou que $40 \%$ a $80 \%$ dos pacientes sofrerão fadiga importante antes, durante ou, até mesmo, após o tratamento quimioterápico ${ }^{22}$. Além disso, esse sintoma se correlaciona com uma piora da QV desses pacientes ${ }^{23}$.

No início do tratamento, os sintomas náuseas/ vômitos mostraram baixos valores médios dos escores $(9,5 \%)$, o que era esperado; pois as pacientes não haviam recebido os quimioterápicos, os quais têm potencial emetogênico. Contudo, houve um aumento acentuado dos valores médios no terceiro ciclo $(42,9 \%)$ indicando a intensificação desses sintomas durante o tratamento quimioterápico; porém, no último ciclo analisado, ocorreu uma redução da média $(33,3 \%)$, representando diminuição desses sintomas. A dor, que já havia sido identificada em $20 \%$ das mulheres na primeira avaliação, mostrou aumento dos seus valores médios no segundo momento (31\%) e no terceiro momento $(33,3 \%)$; ou seja, intensificação desse sintoma, levando a ser considerado um forte indicador para influenciar negativamente a QV das pacientes, podendo impactar negativamente a QV. Esse achado deve ser utilizado como importante avaliador na prática clínica, uma vez que o paciente deverá ser assistido quanto às novas necessidades e adaptaçóes, sendo fundamental a intervenção do profissional de saúde. As repercussóes da dor sobre a capacidade de viver plenamente trazem impacto sobre o estado de saúde e prejuízos à $\mathrm{QV}$, devendo portanto ser considerado e cuidadosamente analisado.

Diante do sintoma insônia, verifica-se sua ocorrência $(23,8 \%)$ já no início do tratamento, o que foi intensificado nos ciclos subsequentes, apresentando, na avaliação do terceiro ciclo, valor médio do escore $(35,7 \%)$ e um aumento de quase $50 \%$ no último ciclo $(45,2 \%)$ em relação ao primeiro ciclo; traduzindo piora da QV. $\mathrm{Na}$ prática clínica, são bastante comuns os relatos de insônia por pacientes com diagnóstico recente de câncer, ou mesmo aqueles em início de tratamento, em que traduzem, por meio desse sintoma, sentimentos como ansiedade, medo, revolta.

Quanto à perda de apetite, também se verificou um aumento importante dos valores médios dos escores desse sintoma durante o curso do tratamento. No primeiro ciclo do tratamento, o valor médio do escore apresentado foi de 9,5\%, aumentando para 40,5\%, no terceiro ciclo; e, deste, para $45,2 \%$ no último ciclo. Esses resultados mostram que o tratamento intensificou a perda de apetite nas pacientes 
Tabela 2. Valores médios dos domínios do EORTC-30 entre as 14 participantes da pesquisa submetidas à quimioterapia adjuvante. Uberlândia (MG), 2011

\begin{tabular}{|c|c|c|c|c|}
\hline Domínios EORT C30 & Média & DP* & Mínimo & Máximo \\
\hline Função física 1 & 82,4 & 12,1 & 57 & 100 \\
\hline Função física 2 & 71,4 & 14,5 & 43 & 95 \\
\hline Função física 3 & 68,8 & 14,0 & 48 & 90 \\
\hline Função emocional 1 & 66,9 & 22,1 & 42 & 100 \\
\hline Função emocional 2 & 68,4 & 18,7 & 33 & 92 \\
\hline Função emocional 3 & 65,0 & 31,1 & 0 & 100 \\
\hline Função cognitiva 1 & 79,6 & 24,7 & 33 & 100 \\
\hline Função cognitiva 2 & 69,2 & 12,7 & 50 & 100 \\
\hline Função cognitiva 3 & 58,4 & 29,0 & 17 & 100 \\
\hline Função social 1 & 89,3 & 16,8 & 50 & 100 \\
\hline Função social 2 & 100 & 0 & 100 & 100 \\
\hline Função social 3 & 86,9 & 29,3 & 0 & 100 \\
\hline Fadiga 1 & 27,4 & 20,3 & 0 & 83 \\
\hline Fadiga 2 & 40,5 & 21,4 & 0 & 83 \\
\hline Fadiga 3 & 41,7 & 29,1 & 0 & 83 \\
\hline Náuseas/vômitos 1 & 9,5 & 26,7 & 0 & 100 \\
\hline Náuseas/vômitos 2 & 42,9 & 29,8 & 0 & 100 \\
\hline Náuseas/vômitos 3 & 33,3 & 22,6 & 0 & 67 \\
\hline Dor 1 & 20,2 & 25,5 & 0 & 67 \\
\hline Dor 2 & 31,0 & 33,9 & 0 & 100 \\
\hline Dor 3 & 33,3 & 28,5 & 0 & 83 \\
\hline Dispneia 1 & 0 & 0 & 0 & 33 \\
\hline Dispneia 2 & 4,8 & 12,1 & 0 & 33 \\
\hline Dispneia 3 & 4,8 & 12,1 & 0 & 100 \\
\hline Insônia 1 & 23,8 & 35,6 & 0 & 100 \\
\hline Insônia 2 & 35,7 & 33,2 & 0 & 100 \\
\hline Insônia 3 & 45,2 & 33,6 & 0 & 100 \\
\hline Perda de apetite 1 & 9,5 & 20,4 & 0 & 67 \\
\hline Perda de apetite 2 & 40,5 & 35,0 & 0 & 100 \\
\hline Perda de apetite 3 & 45,2 & 33,6 & 0 & 100 \\
\hline Constipação 1 & 7,1 & 26,7 & 0 & 100 \\
\hline Constipação 2 & 23,8 & 35,6 & 0 & 100 \\
\hline Constipação 3 & 19,1 & 28,4 & 0 & 100 \\
\hline Diarreia 1 & 0 & 0 & 0 & 0 \\
\hline Diarreia 2 & 11,9 & 21,1 & 0 & 67 \\
\hline Diarreia 3 & 21,4 & 21,1 & 0 & 67 \\
\hline Dificuldade financeira 1 & 23,8 & 35,6 & 0 & 100 \\
\hline Dificuldade financeira 2 & 9,5 & 15,6 & 0 & 33 \\
\hline Dificuldade financeira 3 & 29,7 & 37,1 & 0 & 100 \\
\hline$M G S / Q V 1 * *$ & 83,3 & 10,3 & 67 & 100 \\
\hline MGS/QV2** & 80,4 & 11,1 & 67 & 100 \\
\hline MGS/QV3** & 80,9 & 16,5 & 67 & 100 \\
\hline
\end{tabular}

* DP: Desvio-Padrão; ** MGS/QV: Média Geral de Saúde/Qualidade de Vida.

em tratamento. Há que se considerar que a associação entre os diferentes sintomas relacionados ao tratamento quimioterápico e à própria doença podem desencadear uma sobreposição de sintomas e ainda a intensificação de um ou outro; dessa forma, é possível inferir que a ocorrência de náuseas e vômitos tem normalmente sido associada à perda do apetite, o que corrobora a perda de peso, déficits sanguíneos e consequentemente dificuldades para manter o tratamento de maneira efetiva, como planejado inicialmente. Frente a essa situação, foram 
encontradas muitas pacientes com relatos de tristeza, insônia e dificuldades na manutençáo de suas atividades. Em relação à constipação intestinal, nota-se que, no primeiro momento avaliado, esse sintoma já estava presente $(7,1 \%)$; porém, triplicou no segundo momento $(23,8 \%)$, indicando aumento de incidência desse sintoma no terceiro ciclo de tratamento, mas mostrou-se diminuído no último ciclo $(19,1 \%)$, significando melhora no hábito intestinal. Entre os quimioterápicos utilizados, ressalta-se que alguns deles podem levar a alteraçóes do hábito intestinal, o que é considerado pelas pacientes como uma alteraçáo extremamente incômoda e que traz preocupação. A diminuição de ocorrência da constipação tem sido observada na prática clínica quando as pacientes apresentam adesáo às orientaçóes para que fiquem atentas ao início do sintoma e corrijam as alteraçóes por meio de mudanças nos hábitos alimentares; essa prática tem reduzido significativamente esse sintoma e trazido, portanto, melhora na QV.

Finalmente, os resultados mostram que as mulheres do estudo não apresentaram diarreia no início da quimioterapia, sendo os valores médios dos escores desse sintoma no terceiro $11,9 \%$ e no último ciclo $21,4 \%$. Tal efeito era esperado, visto que a maioria das pacientes deste estudo foi tratada sob o esquema FAC, no qual a incidência de diarreia em pacientes tratados com esses quimioterápicos é baixa ${ }^{7}$.

Quanto à dificuldade financeira, observa-se que houve, na segunda avaliação, uma elevaçáo bastante significativa, tendo em vista que a maioria das pacientes normalmente não tem continuidade em atividades remuneradas durante o percurso do tratamento oncológico, seja por dificuldades físicas, ou mesmo pela necessidade de deslocamento para o tratamento; assim, a melhora das condiçôes só ocorre após a finalização ou no período final, em que já é possível começar a retomar o trabalho e, consequentemente, conseguir o retorno financeiro. Essa dificuldade traz impactos negativos para a $\mathrm{QV}$, uma vez que limita o poder aquisitivo, trazendo, muitas vezes, dificuldades, inclusive, para o sustento básico de alimentaçáo e moradia.

Frente a diversas análises realizadas com pacientes com câncer, submetidos a tratamento quimioterápico, observase que eles podem apresentar uma variedade de efeitos colaterais como dor, náusea, vômitos, queda de cabelos, alteração de peso, fadiga, ansiedade, obstipação intestinal, diarreia e outros. No entanto, o efeito colateral mais comumente relatado tem sido a fadiga ${ }^{20}$. Nossos resultados, portanto, corroboram esse achado da literatura, podendo ser notado que esse sintoma, avaliado por meio do EORTC QLQ-30, apresentou média e desvio-padrão aumentados entre os ciclos de quimioterapia aqui estudados.

Para avaliar a capacidade física e benefícios à saúde de um programa multidimensional para pacientes com câncer submetidos à quimioterapia, pesquisadores utilizaram os instrumentos EORTC QLQ-30, para avaliar QV; o SF 36, para avaliar o bem-estar geral e o Hospital Anxiety and Depression Scale (HAD), para avaliar bem-estar psicológico. Neste estudo, a QV e as avaliaçóes gerais do bem-estar indicaram melhoria em diversas medidas, mas sem alcance de significância. Os autores concluíram que um programa de exercício, que combine atividades físicas de intensidades elevada e baixa, pode ser usado para impedir ou/e minimizar a inatividade física, a fadiga e a perda muscular e de energia nos pacientes com câncer submetidos à quimioterapia ${ }^{24}$.

No domínio estado geral de saúde/QV , a alteração foi discreta durante os ciclos quimioterápicos analisados, em que, no primeiro ciclo, o valor médio do escore apresentado foi $83,3 \%$, ocorrendo pequena redução no terceiro ciclo $(80,4 \%)$ e último ciclo $(80,9 \%)$. Nota-se que, apesar dos vários efeitos colaterais e alterações quanto à função física, provocados pelos esquemas quimioterápicos, as avaliaçóes referidas pelas pacientes, para a MGS/QV, foram positivas, uma vez que, em todos os momentos de análise, os valores médios dos escores se mostraram elevados.

É importante ressaltar que as mudanças ocorridas na dinâmica de atendimento médico, durante o período da coleta de dados, no local de desenvolvimento do estudo, impactaram negativamente no número de pacientes que poderiam ser incluídos nesta pesquisa, náo sendo possível incluir o número previsto no projeto inicial. Apesar dessa limitaçáo, considera-se que os resultados encontrados sejam relevantes para a divulgação em meio à comunidade científica.

\section{CONCLUSÃO}

Quanto à caracterização da população estudada, encontrou-se um grupo de mulheres com características semelhantes às de outros estudos que buscaram caracterizar a população e avaliar a QV de pacientes com câncer de mama submetidas à quimioterapia. Nesta busca bibliográfica, nenhum estudo apresentou como particularidade estudar pacientes em tratamento adjuvante, o que ressalta a importância do presente estudo. Ressalta-se ainda a semelhança do grupo estudado quanto às características socioeconômicas, tendo em vista que populaçóes como essa fazem parte de pacientes tratados pelo sistema público de saúde, o qual atende a grande maioria da populaçấo brasileira; essa particularidade torna possível a aplicabilidade dos resultados à população de forma geral.

A despeito de todos os benefícios cientificamente comprovados do tratamento quimioterápico adjuvante, para pacientes com diagnóstico de câncer de mama, observa-se, no conjunto de resultados desta pesquisa, o impacto negativo sobre a $\mathrm{QV}$, desde o início do tratamento até o ultimo ciclo de quimioterapia. Ao 
avaliar isoladamente os domínios função física, emocional e cognitiva, verificou-se piora na QV do início para o final do tratamento. Em relação aos aspectos físicos, todos apresentaram piora da QV.

$\mathrm{Na}$ avaliação da MGS/QV, houve especial divergência quando comparados os resultados dos quatro domínios e da escala de sintomas com a avaliação da MGS/QV, uma vez que, contrariamente ao esperado, foi verificada avaliação positiva da MGS/QV. Para esse resultado, foi inferido que a possibilidade de resultados positivos e a chance de cura, mediante a finalização do tratamento quimioterápico, são possibilidades apresentadas e fatores motivadores, que conduzem as mulheres à sua utilização, enfrentando todas as suas dificuldades, inclusive aquelas que podem interferir na QV.

\section{CONTRIBUIÇÕES}

Audir Giordano C. Guimarães e Anna Cláudia Y. dos Anjos contribuíram na concepção e planejamento do projeto de pesquisa; na obtenção e/ou análise e interpretação dos dados; na redação e na revisão crítica.

\section{Declaração de Conflito de Interesses: Nada a Declarar.}

\section{REFERÊNCIAS}

1. Instituto Nacional de Câncer (Brasil). O que é câncer? Rio de Janeiro: INCA; 2008.Disponível em: http:// wwwl.inca.gov.br/conteudo_view.asp?id=322. Acesso em: 22 abr 2009.

2. Zandonai AP, Cardozo FMC, Nieto ING, Sawada NO. Qualidade de vida nos pacientes oncologicos: revisão integrativa da literatura latino americana. Revista eletrônica de enfermagem 2010; 12(3): 554 - 61.

3. Mathews CF. Diagnóstico do câncer de mama na vida de uma mulher. Revista brasileira de mastologia 2002;p36-48.

4. Ramos JJ. Oncologia clínica. 2. ed. São Paulo: Sarvier, 2002.

5. Bonassa EMA. Enfermagem em terapêutica oncológica. 3. ed. São Paulo: Atheneu, 2005.

6. Sales CA, Paiva L, Scandiuzzi D, Anjos ACY. Qualidade de vida de mulheres tratadas de câncer de mama: funcionamento social. Revista brasileira de cancerologia 2001;47 (3):263-72.

7. Bech AG. Qualidade de vida do paciente oncologico. Disponível em: <http:// www.alss.org/es/Acts/27-BR. Doc> Acesso em 17 ago 2011.

8. Markluf ASD, Dias RC, Barra AA. Avaliação da qualidade de vida em mulheres com câncer da mama. Revista brasileira de cancerologia 2006; 52(1): 49 - 58 .

9. Lotti RCB, Barra AA, Dias RC, Makluf ASD. Impacto do câncer de mama na qualidade de vida. Revista brasileira de cancerologia 2008; 54(4): 367-71.
10. Heywangk SH, Schreer I, Dershaw DD, Frasson A. A paciente jovem. In: Aguillar V, Bauab S, Maranhão N. Mama - diagnóstico por imagem. 1. ed. Rio de Janeiro: Revinter, 1999. p.391-403.

11. Oliveira OLR, Carneiro PCA, Sales Filho R, Oliveira DP. Câncer de mama em mulheres jovens: aspectos epidemiológicos. Revista brasileira de cancerologia 1999; 36(2): 6-9. Disponível em: http://www.rbscancer.com. $\mathrm{br} / \mathrm{rsbc} / 5$ artigo3asp?nrev=No5. Acesso 15 ago 2011.

12. Baségio DL, Koch HA. Formas de diagnóstico do câncer de mama na mulher gaúcha. Revista brasileira de mastologia 1998;8: 64 - 71 .

13. Bairros FS, Meneghel SN, Olinto MTA. Citopatologia e exame de mama: desigualdade de acesso para mulheres negras no sul do Brasil. Epidemiologia e serviços de saúde: revista do Sistema Unico de Saúde do Brasil 2008 abr./ jun.;17(2):123:138-41.

14. Rosa TEC, Benício MHA, Latorre MRDO, Ramos LR. Fatores determinantes da capacidade funcional entre idosos. Rev Saude Publica 2003 fev. 37(1): 40 - 8.

15. Janz NK, Mujahid M, Lantz PM, Fagerlin A, Salem B, Morrow M, et al. Population based study of the relationship of treatment and sociodemographics on quality of life for ealy stage breast cancer. Qual Life Res. 2005 Aug;14(6):1467-79.

16.IBGE. Estatísticas do registro civil 2010. Disponível em: http://www.ibge.gov.br/home/estatistica/populacao/ registrocivil/2010/rc2010.pdf. v. 37. Acesso em 23 nov.2011.

17. Forattini OP. Qualidade de vida e meio urbano: a cidade de São Paulo, Brasil. Rev Saude Publica 1991; 25(2): 75-86.

18. Aranibar HG, Ramirez AL, Alvarez M. Quimioterapia adjuvante em axila comprometida. Es necessário planificar esquemas distintos según cantidad de nódulos metastásicos? Revista chilena de cirurgia. 2005 febr.; 57 (1): 81 - 8 .

19. Jong N, Candel MJJM, Shouten HC, Abu - Saad HH, Courtens HM. Prevalence and course of fatigue in breast cancer patients receiving adjuvant chemotherapy. Annals of oncology 2004; 15: 896-905.

20. National Cancer Institute. Disponível em: http://www. cancer.gov/ Acesso 23 de set. 2011.

21. Silva CB, Albuquerque V, Leite J. Qualidade de vida em pacientes portadoras de neoplasia mamária submetida a tratamentos quimioterápicos. Revista brasileira de cancerologia 2010; 56(2): 227 - 36

22. Hofman M, Ryan JL, Fiqueroa MCD, Jean PP, Morrow GR. Cancer related fatique: the scale of the problem. Oncologist 2007;12 suppl 1:4 - 10

23. Novais MAB, Glasberg J, Chicoli FA, Canavezzi AZ, Xavier WC, Samano EST, et al. Fadiga, depressão e qualidade de vida em pacientes com câncer de mama submetidas a radioterapia (online). Disponível em: http//www.saudebrasilnet.com.br/prêmios/oncologia/ premio2/trabalhos/saúde 2008.pdf

24. Markluf ASD, Dias RC, Barra AA. Avaliação da qualidade de vida em mulheres com câncer da mama. Revista brasileira de cancerologia 2006; 52(1): $49-58$. 


\section{ANEXOS}

ANEXO 1

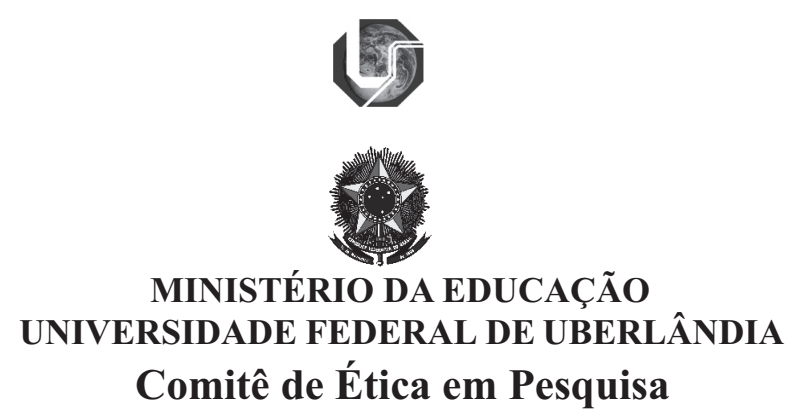

\section{QUESTIONÁRIO A}

Prontuário:

Diagnóstico médico:

Período do tratamento:

Medicação prescrita:

\begin{tabular}{|l|}
\hline 1- DADOS DE IDENTIFICAÇÃO \\
\hline Nome (iniciais): \\
\hline Data de nascimento: \\
\hline Peso (kg): \\
\hline Cor da pele: \\
\hline Profissão: \\
\hline No momento esta trabalhando: $\square$ Sim $\square$ Não \\
\hline Estado civil: \\
\hline Grau de instrução: \\
\hline Renda familiar: \\
\hline Procedência: \\
\hline 2- INFORMAÇÕES CLINICAS E TERAPÊUTICAS \\
\hline Estadiamento: \\
\hline Esquema terapêutico: \\
\hline Possui outra patologia: $\square$ Sim $\square$ Não \\
Se SIM, qual (is): \\
\\
\\
\hline Efeitos Colaterais: \\
$\square \quad$ Náuseas \\
$\square \quad$ Vômitos \\
$\square \quad$ Constipação \\
$\square \quad$ Diarreia \\
$\square \quad$ Alopecia \\
$\square \quad$ Mucosite \\
$\square \quad$ Fraqueza \\
$\square \quad$ Febre \\
\hline
\end{tabular}




\section{EORTC QLQ-C30 (version 3)}

Gostaríamos de conhecer alguns pormenores sobre si e a sua saúde. Responda você mesmo/a, por favor, a todas as perguntas fazendo um círculo à volta do número que melhor se aplica ao seu caso. Não há respostas certas nem erradas. A informação fornecida é estritamente confidencial.

Escreva as iniciais do seu nome:

A data de nascimento (dia, mês, ano):

A data de hoje (dia, mês, ano):

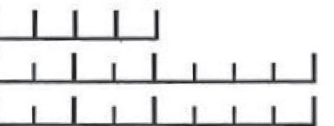

31

1. Custa-Ihe fazer esforços mais violentos, por exemplo carregar um saco de compras pesado ou uma mala?

2. Custa-lhe percorrer uma grande distância a pé?

3. Custa-lhe dar um pequeno passeio a pé, fora de casa?

4. Precisa de ficar na cama ou numa cadeira durante o dia?

5. Precisa que o/a ajudem a comer, a vestir-se, a lavar-se ou a ir à casa de banho?

\section{Durante a última semana :}

6. Sentiu-se limitado/a no seu emprego ou no desempenho das suas actividades diárias?

7. Sentiu-se limitado/a na ocupação habitual dos seus tempos livres ou noutras actividades de laser?

8. Teve falta de ar?

9. Teve dores?

10. Precisou de descansar?

11. Teve dificuldade em dormir?

12. Sentiu-se fraco/a?

13. Teve falta de apetite?

14. Teve enjoos?

15. Vomitou?
Não

\section{Um Bastante Muito pouco}

$\begin{array}{llll}1 & 2 & 3 & 4 \\ 1 & 2 & 3 & 4 \\ 1 & 2 & 3 & 4 \\ 1 & 2 & 3 & 4 \\ 1 & 2 & 3 & 4\end{array}$

Não $\underset{\text { pouco }}{\text { Um Bastante Muito }}$

$\begin{array}{lllll}1 & 2 & 3 & 4\end{array}$

$\begin{array}{llll}1 & 2 & 3 & 4 \\ 1 & 2 & 3 & 4 \\ 1 & 2 & 3 & 4 \\ 1 & 2 & 3 & 4 \\ 1 & 2 & 3 & 4 \\ 1 & 2 & 3 & 4 \\ 1 & 2 & 3 & 4 \\ 1 & 2 & 3 & 4 \\ 1 & 2 & 3 & 4\end{array}$

Por favor, passe à página seguinte 
Durante a última semana :

16. Teve prisão de ventre?

17. Teve diarreia?

18. Sentiu-se cansado/a?

19. As dores perturbaram as suas actividades diárias?

20. Teve dificuldade em concentrar-se, por exemplo, para ler o jornal ou ver televisão?

21. Sentiu-se tenso/a?

22. Teve preocupações?

23. Sentiu-se irritável?

24. Sentiu-se deprimido/a?

25. Teve dificuldade em lembrar-se das coisas?

26. O seu estado físico ou tratamento médico interferiram na sua vida familiar?

27. O seu estado físico ou tratamento médico interferiram na sua actividade $\underline{\underline{s o c i a l}}$ ?

28. O seu estado físico ou tratamento médico causaram-Ihe problemas de ordem financeira?

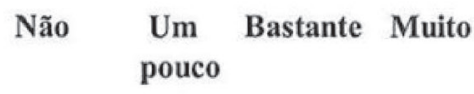

$\begin{array}{llll}1 & 2 & 3 & 4 \\ 1 & 2 & 3 & 4 \\ 1 & 2 & 3 & 4 \\ 1 & 2 & 3 & 4\end{array}$

$\begin{array}{llll}1 & 2 & 3 & 4\end{array}$

$\begin{array}{llll}1 & 2 & 3 & 4\end{array}$

$\begin{array}{llll}1 & 2 & 3 & 4\end{array}$

$\begin{array}{llll}1 & 2 & 3 & 4\end{array}$

$\begin{array}{llll}1 & 2 & 3 & 4\end{array}$

$\begin{array}{llll}1 & 2 & 3 & 4\end{array}$

$\begin{array}{llll}1 & 2 & 3 & 4\end{array}$

$\begin{array}{llll}1 & 2 & 3 & 4\end{array}$

$\begin{array}{llll}1 & 2 & 3 & 4\end{array}$

Nas perguntas que se seguem faça um círculo à volta do número, entre $1 \mathrm{e} 7$, que melhor se aplica ao seu caso

29. Como classificaria a sua saúde em geral durante a última semana?
1
2
3
4
5
6
7

Péssima

Óptima

30. Como classificaria a sua qualidade de vida global durante a última semana?
1
2
3
4
5
6
7

Péssima

Óptima

O Copyright 1995 EORTC Study Group on Quality of Life. Todos os direitos reservados. Version 3.0. 


\section{Abstract}

Introduction: Evaluating Quality of Life (QL) is an important auxiliary in clinical practice. Objective: Evaluate the quality of life (QOL) in women with breast cancer and to characterize them as to socio-demographic aspects during adjuvant chemotherapy. Method: An exploratory descriptive study with a quantitative approach, which used two instruments: one for socio-demographic evaluation and another for QoL (EORTC QLQ-30). The sample consisted of 14 patients treated at the oncology outpatient clinic of a university hospital in Minas Gerais, from April to October 2011.Results: The majority of the population has white skin color $(57.1 \%)$, are married $(64.3 \%)$ and there was a predominance of women with incomplete primary level (35.7\%) and complete secondary level (35.7\%). Monthly family income ranged from 2 to 6 minimum wages (78.6\%). As to origin, $50 \%$ are from Uberlandia (50\%) and outside Uberlandia (50\%). The therapeutic regimen was predominant use of the FAC (50\%) followed by the CMF scheme (28.6\%). When we evaluated separately the areas of physical, emotional and cognitive function, QoL was worsened from the beginning towards the end of treatment. Regarding the physical aspects, all women had lower QoL. In assessing the extent of overall health /quality of life (QOL/MGS), a slight reduction was observed, even with a positive evaluation of patients throughout the study. Conclusion: The set of sample results showed characteristics similar to other studies, and show that, despite all the scientifically proven benefits of adjuvant chemotherapy, its negative impact on QOL throughout the treatment of cancer patients breast cancer treated with adjuvant chemotherapy can be noted. Key words: Quality of Life; Breast Neoplasms; Women; Chemotherapy, Adjuvant; Socioeconomic Factors; Population Surveys

\section{Resumen}

Introducción: La evaluación de la calidad de vida $(\mathrm{CdV})$ es importante auxiliar en la práctica clínica. Objetivo: Evaluar la $\mathrm{CdV}$ en mujeres con cáncer de mama y sus características en cuanto a los aspectos sociodemográficos durante el tratamiento de la quimioterapia adyuvante. Método: Estudio exploratorio, descriptivo, con abordaje cuantitativo. Han sido utilizados dos herramientas: una para la evaluación sociodemográfica y otra para la evaluación de la CdV (EORTC QLQ-C30). La muestra se basó en 14 pacientes, con atendimiento en consulta de oncología de un hospital universitario de Minas Gerais, desde abril hasta octubre del 2011. Resultados: El estudio ha demostrado que la mayoría de la población es de color blanca $(57,1 \%)$, está casada $(64,3 \%)$, hay predominancia de mujeres con Educación General Básica incompleta $(35,7 \%)$ y instituto completo $(35,7 \%)$. Los ingresos familiares mensuales han sido de dos a seis rendas mínimas (78,6\%). En cuanto a la procedencia, el (50\%) son de Uberlância y el $(50 \%)$ de fuera de la ciudad. Acerca de la metodología terapéutica, ha sido de predominancia la utilización de la FAC (50\%), seguido de la metodología de CMF (28,6\%). Al evaluar de manera aislada las funciones física, emocional y cognitiva, se ha verificado un empeoramiento en la $\mathrm{CdV}$ desde el inicio hasta el final de tratamiento. En cuanto a los aspectos físicos, todas las mujeres han demostrado también disminución en la calidad de vida. En la evaluación calidad de vida/medidas de seguridad sanitaria (CDV/MGS) se observó una leve reducción, incluso con una evaluación positiva de los pacientes durante el estudio. Conclusión: El conjunto de resultados de la muestra presentó características similares a otros estudios, además de poner en evidencia, que a pesar de todos los beneficios comprobados de manera científica de la quimioterapia adyuvante, se ha observado su impacto negativo sobre la calidad de vida, durante todo el periodo del tratamiento.

Palabras clave: Calidad de Vida; Neoplasias de la Mama; Mujeres; Quimioterapia Adyuvante; Factores Socioeconômicos; Encuestas Demográficas 